\title{
Sintomas depressivos: estudo exploratório em jovens vestibulandos para o curso de medicina no interior da Amazônia
}

\author{
Depressive symptoms: exploratory study in young vestibulands for the medicine course \\ inside the Amazon
Síntomas depresivos: estudio exploratorio en jóvenes vestibulandos para el curso de medicina dentro de la Amazonia

Caroline Sayuri Lustosa Sato ${ }^{1}$, Rafaela Martins Dourado Gonçalves ${ }^{1}$, Ana Emília Gomes Macêdo ${ }^{1,2}$, Caroline Gomes Macêdo ${ }^{3 *}$.

\begin{abstract}
RESUMO
Objetivo: Avaliar a presença de sintomas depressivos em pré-vestibulandos para o curso de medicina, no município de Santarém-Pará. Métodos: Realizou-se um estudo descritivo, de análise quantitativa e transversal, com a aplicação de dois questionários a 51 estudantes de dois cursos de pré-vestibular da cidade, sendo um para avaliação socioeconômica e outro, o BDI, para a identificação da presença de sintomas depressivos e classificação de seu grau. Resultados: O perfil prevalente encontrado nesta pesquisa foi de mulheres, solteira, pardas, entre 18 aos 20 anos, com renda familiar mensal média de 5 ou mais salários mínimos. Quanto à prevalência de sintomas depressivos, 49,02\% dos participantes possuíam sintomas depressivos moderados a graves, sendo estes mais frequentes em mulheres jovens, solteiras e que estavam a mais de 2 anos no curso pré-vestibular. 11 participantes possuíam comorbidades prévias relatadas, sendo a mais prevalente na pesquisa o Transtorno de Ansiedade Generalizado, seguido por atopias. Conclusão: Espera-se que os resultados apresentados estimulem novas pesquisas dentro do tema estudado, diminuindo o estigma ao redor das doenças psiquiátricas, assim como aumente o investimento de instituições de ensino na manutenção da saúde mental dos seus estudantes.
\end{abstract}

Palavras-chave: Depressão, Saúde mental, Estudantes, Medicina.

\begin{abstract}
Objective: Evaluate the presence of depressive symptoms in students taking the entrance exam for medical school in Santarém-Pará. Methods: A descriptive and cross-sectional study was carried, with quantitative analyses and the application of questionnaires to 51 students from university entrance exam courses in the city. Two questionnaires where applied, one for socioeconomic evaluation and another to identify de presence of depressive symptoms and to rate their grade, using Beck's Depression Inventory. Results: The prevalent profile found in this research consisted of single, brown, women, between 18-20 years old, with an average monthly family income of 5 or more minimum wages. As for the prevalence of depressive symptoms, $49.02 \%$ of the participants had moderate to severe depressive symptoms, which were more frequent in young, single women who had been taking the university entrance exam for more than 2 years. 11 participants had previously reported comorbidities, the most prevalent in the research being Generalized Anxiety Disorder, followed by atopy. Conclusion: It is expected that the results presented will stimulate further research within the studied theme, reducing the stigma surrounding psychiatric illnesses, as well as increasing the investment of educational institutions in maintaining the mental health of their students.
\end{abstract}

Key words: Depression, Mental health, Students, Medicine.

\section{RESUMEN}

Objetivo: Evaluar la presencia de síntomas depresivos en estudiantes preuniversitarios de la carrera de medicina, en la ciudad de Santarém-Pará. Métodos: Se realizó un estudio descriptivo, cuantitativo y

1 Universidade do Estado do Pará (UEPA), Santarém - PA.

2 Universidade Federal do Oeste do Pará (UFOPA), Santarém - PA.

3 Universidade Federal do Pará (UFPA), Belém - PA. *E-mail: carolgomesmacedo@hotmail.com c

SUBMETIDO EM: 1/2022

ACEITO EM: 2/2022

PUBLICADO EM: $3 / 2022$ 
transversal, con la aplicación de dos cuestionarios a 51 estudiantes de dos cursos preuniversitarios de la ciudad, uno de evaluación socioeconómica y el otro, el BDI, para identificar la presencia de los síntomas depresivos y clasificación de su grado. Resultados: El perfil predominante encontrado en esta investigación fue el de mujeres solteras, mestizas, con edades entre 18 y 20 años, con ingreso familiar promedio mensual de 5 salarios mínimos o más. En cuanto a la prevalencia de síntomas depresivos, el 49,02\% de los participantes presentaron síntomas depresivos moderados a severos, siendo más frecuentes en mujeres jóvenes, solteras y con más de 2 años de estudios universitarios. 11 participantes habían informado de comorbilidades previas, siendo la más prevalente en la encuesta el Trastorno de Ansiedad Generalizada, seguida de la atopia. Conclusión: Se espera que los resultados presentados estimulen más investigaciones dentro del tema estudiado, reduciendo el estigma en torno a las enfermedades psiquiátricas, así como aumentando la inversión de las instituciones educativas en el mantenimiento de la salud mental de sus estudiantes.

Palabras clave: Depresión, Salud mental, Estudiantes, Medicina.

\section{INTRODUÇÃO}

A depressão é um transtorno mental que atinge uma parcela significativa da população mundial, com estimativa de 300 milhões de indivíduos af etados (ORGANIZAÇÃO PAN-AMERICANA DE SAÚDE (OPAS), 2018a).

No Brasil, de acordo com a Pesquisa Nacional de Saúde de 2013, realizada pelo Instituto Brasileiro de Geografia e Estatística (IBGE), atualmente mais de 11 milhões de pessoas possuem o diagnóstico de depressão, o que coloca o país em primeiro lugar nos índices da América Latina (IBGE, 2014).

A Organização Mundial da Saúde define adolescência o período que vai de 10 a 19 anos e caracteriza jovens os indivíduos de 15 a 24 anos (MINISTÉRIO DA SAÚDE, 2017). Estudos demonstram que cerca de $50 \%$ dos transtornos mentais têm início ainda na adolescência (OPAS, 2018b).

No entanto, o reconhecimento da depressão como uma psicopatologia presente em adolescentes e jovens é muito recente, tendo recebido maior atenção devido ao aumento dos casos clínicos diagnostic ados, a sua relação direta com outras doenças, como doenças cardiovasculares, metabólicas e hipertensão, e com comportamentos autodestrutivos (SCHNEIDER ACN e RAMIRES VRR, 2007; BIAZUS CB e RAMIRES VRR, 2012; BOING AF, et al., 2012). Além disso, esse transtorno mental está associado ao aumento da incidência de suicídio entre jovens e adultos na faixa etária de 15 a 29 anos (WORLD HEALTH ORGANIZATION, 2013).

Sabe-se que o período que antecede o vestibular pode ser extremamente ansiogênico e estressante, causando impactos diretos na qualidade de vida do vestibulando. O estresse ao qual esses estudantes estão submetidos diariamente podem levar ao aparecimento de sintomas físicos e psicológicos, sendo esses últimos os mais evidentes (SANTOS MA, et al., 2016).

Isso se torna preocupante, uma vez que o estresse psicológico, durante períodos prolongados, está diretamente associado ao surgimento de quadros de depressão, ansiedade, desânimo e apatia (LIPP MEN, 2003). Logo, a identificação precoce de sinais e sintomas depressivos é essencial para a obtenção de um sucesso terapêutico, evitando possíveis complicações e um aumento dos gastos públicos em saúde (BAHLS SC e BAHLS FRC, 2002; LIMA GMPA, et al., 2018).

Diante disso, esse estudo teve como objetivo avaliar a presença de sintomas depressivo em prévestibulando para o curso de medicina, no município de Santarém-Pará, assim como classificar o grau dos sintomas depressivos (nenhum, leve, moderada e grave), identificar possíveis fatores associados ao surgimento desses sintomas e caracterizar o perfil socioeconômico desses estudantes.

\section{MÉTODOS}

Este estudo foi caracterizado como descritivo, de análise quantitativa e transversal, no qual foi feita a análise de dados coletados durante fevereiro de 2019 a março de 2020. 


\section{Local de pesquisa, população e amostra}

A pesquisa foi realizada em dois cursos pré-vestibulares para Medicina, na cidade de Santarém, no estado do Pará. A amostra total da pesquisa consistiu em 51 participantes, sendo que a seleção dessa amostra aconteceu por participação voluntária dos estudantes, os quais responderam aos convites realizados dentro das salas de aula pelas pesquisadoras. A população participante deste trabalho foi homens e mulheres maiores de 18 anos, matriculados no curso preparatório pré-vestibular para medicina durante o primeiro semestre de 2019 e de 2020, que tenham assinado o Termo de Consentimento Livre e Esclarecido e que não apresentem algum tipo de dificuldade cognitiva que os impossibilite de compreender e responder os questionários.

\section{Questionários aplicados}

Foram aplicados dois questionários aos participantes, sendo um referente aos dados socioeconômicos e outro referente à identificação dos sintomas depressivos e seu grau, no qual foi utilizado o Beck Depression Inventory (BDI-I). O questionário socioeconômico identificava dados acerca de: idade, sexo, gênero, renda familiar, local de proveniência, local de moradia, tempo de curso preparatório, existência de apoio familiar, realização de graduações anteriores completas ou incompletas, ocupação atual, presença de comorbidades, acompanhamento psicológico ou psiquiátrico e diagnóstico de transtornos de humor.

\section{Análise de dados}

Os dados coletados foram organizados e armazenados em tabelas, com o auxílio do software Microsoft $\circledast$ Excel e, posteriormente, analisados. A classificação dos sintomas foi realizada segundo do Inventário de Beck I, o qual possui 21 afirmações e possui pontuação variando de 0 a 63 pontos, demonstrando assim, a presença e severidade de sintomas depressivos. A partir do Score Total (ST) obtido, os participantes foram divididos em quatro grupos de acordo com a severidade de seus sintomas, sendo eles: depressão mínima (SC: 0-13), depressão ligeira (SC:14-19), depressão moderada (SC: 20-28) e depressão severa (SC: 29-63) (MELO CMI, et al. 2021).

\section{Aspectos éticos}

Seguindo a resolução 466/2012 instituída pelo Conselho Nacional de Saúde, a pesquisa foi desenvolvida de acordo com as normas vigentes, cumprindo com os preceitos éticos estabelecidos, assegurando a veracidade e o sigilo das informações aqui contidas, assim como o respeito à privacidade do participante. Este estudo foi aprovado pelo Comitê de Ética em Pesquisa com Seres Humanos da Universidade do Estado do Pará, sob CAAE: 08508819.6.0000.5168.

\section{RESULTADOS}

Traçou-se o perfil epidemiológico dos estudantes segundo variáveis socioeconômicas, tais como sexo, gênero, idade, estado civil, residência, procedência, cor/etnia, religião, profissão e renda familiar mensal. Dos 51 participantes, $52,94 \%$ eram do sexo feminino e $47,06 \%$ do sexo masculino, sendo que destes, $1,96 \%$ não se identificavam com o gênero. Observou-se o predomínio da faixa etária de 18 a 20 anos (76,46\%), do estado civil solteiro $(98,04 \%)$, de pardos $(78,43 \%)$, residentes $(98,03 \%)$ e procedentes $(84,31 \%)$ de Santarém. Somente $15,69 \%$ afirmaram possuir uma profissão, sendo elas: enfermeiro, vendedor, agente de pesquisa e mapeamento do IBGE, agente de viagem, caixa, fotógrafo e policial civil. Quanto a renda familiar mensal, $31,37 \%$ apresentavam mais de 5 salários mínimos, 29,41\% de 2 a 3 salários mínimos, 25,49\% de 3 a 4 salários mínimos e $13,73 \%$ até 1 salário mínimo. Por fim, quando questionados sobre religiosidade, $84,31 \%$ dos indivíduos declararam-se religiosos, sendo que destes, 17,65\% não eram praticantes (Tabela 1). 
Tabela 1- Perfil socioeconômico dos estudantes de pré-vestibular para medicina.

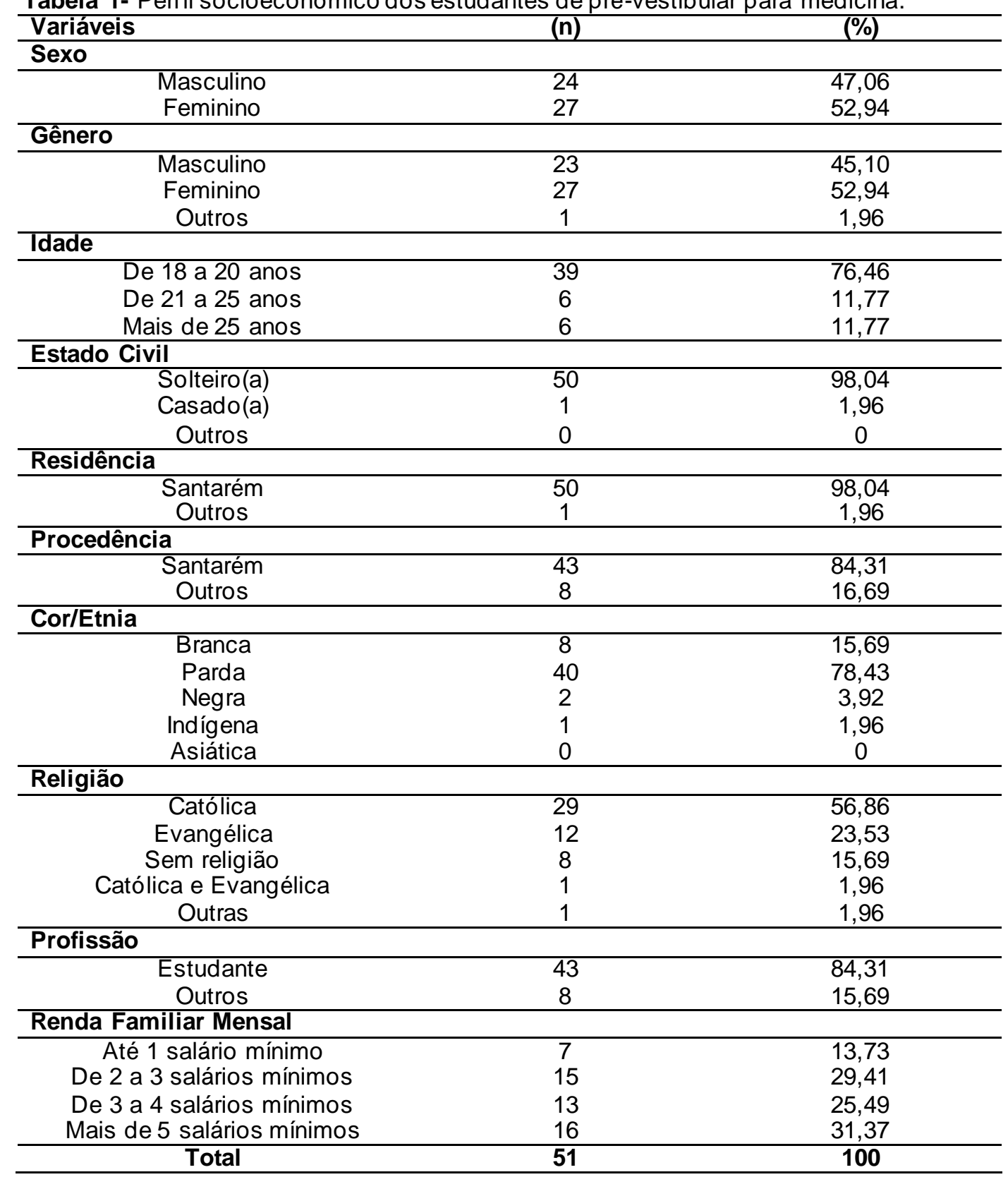

Fonte: Sato CSL, et al., 2022.

Quanto ao nível de escolaridade e o tempo de realização de curso pré-vestibular entre os estudantes, nota-se que apesar de 17,64\% ainda estarem cursando o ensino médio, a maioria dos participantes (76,46\%) já o haviam concluído, sendo que destes, 5,90\% também haviam concluído o ensino superior. Sobre o tempo de realização de curso preparatório para o vestibular, houve maior prevalência na faixa de 2 a 3 anos (62,74\%) e quando questionados sobre a existência de apoio familiar, 96,07\% afirmaram possuí-lo. Além disso, quando analisado os scores obtidos no BDI e correlacionando-os ao tempo de realização de curso preparatório para o vestibular, notou-se que dos 25 indivíduos que apresentaram sintomas depressivos de moderado a grave, 72\% estavam a mais de 2 anos no curso pré-vestibular (Tabela 2).

Foi realizado também a análise de problemas de saúde, relacionados ou não à saúde mental, diagnósticos de transtorno de humor e acompanhamento psicológico/ psiquiátrico entre estudantes dos pré-vestibulares estudados. Quanto à variável sobre problemas de saúde, a maioria dos participantes $(78,43 \%)$ alegou não os possui. As patologias daqueles que responderam positivamente $(21,57 \%)$ dividiam-se em dois grupos: doenças relacionadas a transtornos de humor e outros tipos de doenças. As doenças que foram relatadas 
pelos participantes que não tinham relação com transtornos de humor, foram, em sua maioria, de cunho atópico, como rinites alérgicas, sinusites, ou alergias no geral, de cunho visual, como miopia e astigmatismo, de caráter gastrointestinal, como gastrite, ou de apresentação endócrina, como hipotereoidismo. Quanto às patologias relacionadas a Transtornos de Humor, foi relatada a presença de transtornos de ansiedade, incluindo Transtornos de Ansiedade Generalizada (TAG) e ansiedade crônica, e bipolaridade (Tabela 3).

Tabela 2 - Nível de escolaridade e anos de realização de curso preparatório entre estudantes de pré-vestibular de medicina.

\begin{tabular}{|c|c|c|}
\hline Variáveis & (n) & $(\%)$ \\
\hline \multicolumn{3}{|l|}{ Escolaridade } \\
\hline Ensino Fundamental incompleto & 0 & 0 \\
\hline $\begin{array}{c}\text { Ensino Fundamental completo e Ensino Médio } \\
\text { incompleto }\end{array}$ & 9 & 17,64 \\
\hline $\begin{array}{l}\text { Ensino Médio completo e Ensino Superior } \\
\text { incompleto }\end{array}$ & 39 & 76,46 \\
\hline Ensino Superior completo & 3 & 5,90 \\
\hline \multicolumn{3}{|l|}{ Anos de pré-vestibular } \\
\hline $\begin{array}{c}\text { Até } 1 \text { ano } \\
\text { De } 2 \text { a } 3 \text { anos }\end{array}$ & $\begin{array}{l}13 \\
32\end{array}$ & $\begin{array}{l}25,49 \\
62,74\end{array}$ \\
\hline Mais de 3 anos & 6 & 11,77 \\
\hline Total & 51 & 100 \\
\hline
\end{tabular}

Fonte: Sato CSL, et al., 2022.

Ademais, $86,27 \%$ da amostra negou possuir diagnóstico prévio de transtornos de humor e $84,31 \%$ negaram fazer qualquer tipo de acompanhamento com profissionais da área da saúde mental (Tabela 3).

Tabela 3 - Distribuição de problemas de saúde, diagnósticos de transtorno de humor e acompanhamento psicológico/psiquiátrico entre estudantes de pré-vestibular de medicina.

\begin{tabular}{cccc}
\hline Variáveis & (n) & $(\%)$ \\
\hline Problemas de saúde & & 21,57 \\
& Sim & 11 & \\
\hline Não & 40 & \\
\hline Diagnóstico prévio de Transtornos de humor & & 13,73 \\
Sim & 7 & 86,27 \\
\hline Não & 44 & 15,69 \\
\hline Acompanhamento psicológico/psiquiátrico & & 84,31 \\
\hline Sim & 8 & $\mathbf{1 0 0}$ \\
\hline Não & 43 & \\
\hline Total & $\mathbf{5 1}$ & \\
\hline
\end{tabular}

Fonte: Sato CSL, et al., 2022.

É possível observar a distribuição do Grau de depressão nos participantes de acordo com sua pontuação no Inventário de Beck, observando-se quatro graus: depressão mínima, depressão ligeira, depressão moderada e depressão grave. Dentre os graus, o menos prevalente foi referente à depressão ligeira (15,69\%), enquanto os outros foram relativamente equivalentes entre si, com depressão mínima superior aos outros (35,29\%). Quanto à média de idade daqueles que apresentaram depressão moderada a severa, representando $49,02 \%$ da amostra, essa foi de aproximadamente 20 anos (Tabela 4).

Tabela 4 - Classificação do Grau de depressão entre estudantes de pré-vestibular de medicina, segundo pontuações obtidas no BDI-I.

\begin{tabular}{lcc}
\hline Variáveis & (n) & $(\%)$ \\
\hline Depressão mínima & 18 & 35,29 \\
Depressão ligeira & 8 & 15,69 \\
Depressão moderada & 14 & 27,45 \\
Depressão severa & 11 & 21,57 \\
\hline \multicolumn{1}{c}{ Total } & $\mathbf{5 1}$ & $\mathbf{1 0 0}$ \\
\hline
\end{tabular}

Fonte: Sato CSL, et al., 2022. 
Ao realizarmos a relação entre sintomas depressivos e características socioeconômicas, assim como de tempo de realização de curso pré-vestibular, nota-se que os graus de sintomas depressivos de moderado a severo foram mais frequentes nos estudantes do sexo e gênero feminino (31,38\%), na faixa etária de 18 a 20 anos $(35,3 \%)$, solteiros $(49,02 \%)$, residentes $(47,06 \%)$ e procedentes de Santarém $(41,18 \%)$, que se identificaram como pardos (41,18\%), possuíam religião (41,18\%), não possuíam uma profissão (39,22\%), com renda familiar mensal de 3 a 4 salários $(13,72 \%)$ e que estavam a mais de 2 anos no curso pré-vestibular $(35,29 \%)$ (Tabela 5).

Tabela 5 - Relação entre características sociodemográficas, tempo de pré-vestibular e o BDI-I.

\begin{tabular}{|c|c|c|c|c|}
\hline Variáveis & $\begin{array}{c}\text { Mínima } \\
\mathbf{n}(\%) \\
\end{array}$ & $\frac{\text { Ligeira }}{\text { n (\%) }}$ & $\begin{array}{c}\text { Moderada } \\
\mathbf{n}(\%)\end{array}$ & $\begin{array}{c}\text { Severa } \\
\mathbf{n}(\%)\end{array}$ \\
\hline \multicolumn{5}{|l|}{ Sexo } \\
\hline $\begin{array}{l}\text { Feminino } \\
\text { Masculino }\end{array}$ & $\begin{array}{c}7(13,73) \\
11(21,57) \\
\end{array}$ & $\begin{array}{l}4(7,84) \\
4(7,84) \\
\end{array}$ & $\begin{array}{c}9(17,65) \\
5(9,80) \\
\end{array}$ & $\begin{array}{c}(13,73) \\
4(7,84) \\
\end{array}$ \\
\hline \multicolumn{5}{|l|}{ Gênero } \\
\hline $\begin{array}{l}\text { Feminino } \\
\text { Masculino } \\
\text { Outros }\end{array}$ & $\begin{array}{c}7(13,73) \\
11(21,57) \\
0(0) \\
\end{array}$ & $\begin{array}{c}4(7,84) \\
4(7,84) \\
0(0) \\
\end{array}$ & $\begin{array}{c}9(17,65) \\
5(9,80) \\
0(0) \\
\end{array}$ & $\begin{array}{l}7(13,73) \\
3(5,88) \\
1(1,96) \\
\end{array}$ \\
\hline \multicolumn{5}{|l|}{ Idade } \\
\hline $\begin{array}{l}\text { De } 18 \text { a } 20 \text { anos } \\
\text { De } 21 \text { a } 25 \text { anos } \\
\text { Mais de } 25 \text { anos }\end{array}$ & $\begin{aligned} & 13(25,49) \\
& 2(3,92) \\
& 3(5,88) \\
&\end{aligned}$ & $\begin{array}{c}(15,69) \\
0(0) \\
0(0) \\
\end{array}$ & $\begin{array}{cl}11 & (21,57) \\
2 & (3,92) \\
1 & (1,96) \\
\end{array}$ & $\begin{array}{l}7(13,73) \\
2(3,92) \\
2(3,92) \\
\end{array}$ \\
\hline \multicolumn{5}{|l|}{ Estado civil } \\
\hline $\begin{array}{c}\text { Solteiro } \\
\text { Casado } \\
\text { Outros } \\
\end{array}$ & $\begin{array}{c}17(33,33) \\
1(1,96) \\
0(0) \\
\end{array}$ & $\begin{array}{c}(15,69) \\
0(0) \\
0(0) \\
\end{array}$ & $\begin{array}{c}14(27,45) \\
0(0) \\
0(0) \\
\end{array}$ & $\begin{array}{l}11(21,57) \\
0(0) \\
0(0) \\
\end{array}$ \\
\hline \multicolumn{5}{|l|}{ Residência } \\
\hline $\begin{array}{c}\text { Santarém } \\
\text { Outros }\end{array}$ & $\begin{array}{c}18(35,29) \\
0(0) \\
\end{array}$ & $\begin{array}{c}8(15,69) \\
0(0)\end{array}$ & $\begin{array}{c}13(25,49) \\
1(1,96) \\
\end{array}$ & $\begin{array}{c}11(21,57) \\
0 \\
\end{array}$ \\
\hline \multicolumn{5}{|l|}{ Procedência } \\
\hline $\begin{array}{c}\text { Santarém } \\
\text { Outros }\end{array}$ & $\begin{array}{c}14(27,45) \\
4(7,84)\end{array}$ & $\begin{array}{c}8(15,69) \\
0(0)\end{array}$ & $\begin{array}{c}12(23,53) \\
2(3,92)\end{array}$ & $\begin{array}{c}9(17,65) \\
2(3,92)\end{array}$ \\
\hline \multicolumn{5}{|l|}{ Cor/Etnia } \\
\hline $\begin{array}{c}\text { Branca } \\
\text { Parda } \\
\text { Negra } \\
\text { Indígena } \\
\text { Asiática }\end{array}$ & $\begin{array}{c}4(7,84) \\
14(27,45) \\
0(0) \\
0(0) \\
0(0)\end{array}$ & $\begin{array}{l}2(3,92) \\
5(9,8) \\
1(1,96) \\
0(0) \\
0(0)\end{array}$ & $\begin{aligned} & 0(0) \\
& 13(25,49) \\
& 1(1,96) \\
& 0(0) \\
& 0(0)\end{aligned}$ & $\begin{array}{c}2(3,92) \\
8(15,69) \\
0(0) \\
1(1,96) \\
0\end{array}$ \\
\hline \multicolumn{5}{|l|}{ Religião } \\
\hline $\begin{array}{c}\text { Católica } \\
\text { Evangélica } \\
\text { Católica e evangélica } \\
\text { Sem religião } \\
\text { Outros }\end{array}$ & $\begin{array}{l}9(17,65) \\
4(7,84) \\
1(1,96) \\
3(5,88) \\
1(1,96) \\
\end{array}$ & $\begin{array}{c}3(5,88) \\
4(7,84) \\
0 \\
1(1,96) \\
0 \\
\end{array}$ & $\begin{array}{c}10(19,61) \\
2(3,92) \\
0 \\
2(3,92) \\
0 \\
\end{array}$ & $\begin{array}{c}7(13,73) \\
2(3,92) \\
0 \\
2(3,92) \\
0 \\
\end{array}$ \\
\hline \multicolumn{5}{|l|}{ Profissão } \\
\hline $\begin{array}{c}\text { Estudante } \\
\text { Outros }\end{array}$ & $\begin{array}{c}16(31,37) \\
2(3,92) \\
\end{array}$ & $\begin{array}{c}7(13,73) \\
1(1,96) \\
\end{array}$ & $\begin{array}{c}11(21,57) \\
3(5,88) \\
\end{array}$ & $\begin{array}{c}9(17,65) \\
2(3,92) \\
\end{array}$ \\
\hline \multicolumn{5}{|l|}{ Renda familiar mensal } \\
\hline $\begin{array}{c}\text { Até } 1 \text { salário mínimo } \\
\text { De } 2 \text { a } 3 \text { salários mínimos } \\
\text { De } 3 \text { a } 4 \text { salários mínimos } \\
\text { Mais de } 5 \text { salários mínimos }\end{array}$ & $\begin{array}{c}0(0) \\
6(11,77) \\
6(11,77) \\
6(11,77)\end{array}$ & $\begin{array}{c}0(0) \\
3(5,88) \\
0(0) \\
5(9,80)\end{array}$ & $\begin{array}{l}4(7,84) \\
4(7,84) \\
3(5,88) \\
3(5,88)\end{array}$ & $\begin{array}{l}3(5,88) \\
2(3,92) \\
4(7,84) \\
2(3,92)\end{array}$ \\
\hline \multicolumn{5}{|l|}{ Anos de pré-vestibular } \\
\hline $\begin{array}{c}\text { Até } 1 \text { ano } \\
\text { De } 2 \text { a } 3 \text { anos } \\
\text { Mais de } 3 \text { anos }\end{array}$ & $\begin{array}{c}5(9,80) \\
10(19,61) \\
3(5,88)\end{array}$ & $\begin{array}{c}1(1,96) \\
6(11,77) \\
1(1,96)\end{array}$ & $\begin{aligned} 2 & (3,92) \\
11 & (21,57) \\
1 & (1,96)\end{aligned}$ & $\begin{array}{l}5(9,80) \\
5(9,80) \\
1(1,96)\end{array}$ \\
\hline
\end{tabular}

Fonte: Sato CSL, et al., 2022. 
Dentre os 11 participantes que possuíam patologias distintas do Transtorno Depressivo Maior, foram encontrados 6 diferentes tipos de comorbidades, sendo o mais prevalente O TAG, seguido por atopias, esclerose múltipla, asma e Doença do Refluxo Gastroesofágico (DRGE) e, por fim, hipotireoidismo. As porcentagens indicadas na tabela de relação entre comorbidades prévias relatadas e o BDI-I referem-se à quantos porcento possuem ref erida comorbidade com grau associado dentre os 11 acometidos, sendo o total amostral utilizado para tal tabela de $\mathrm{N}=11$, ao invés da amostra total da pesquisa de 51 participantes (Tabela 6).

Tabela 6- Relação entre comorbidades prévias relatadas e o BDI-I

\begin{tabular}{ccccc}
\hline \multirow{2}{*}{ Variável } & Mínima & Ligeira & Moderada & Severa \\
\cline { 2 - 5 } Comorbidade & $\mathbf{n}(\%)$ & $\mathbf{n}(\%)$ & $\mathbf{n ~ ( \% )}$ & $\mathbf{n}(\%)$ \\
\hline TAG & & & $1(9,09)$ & $2(18,18)$ \\
Atopias & $1(9,09)$ & $1(9,09)$ & $0(0)$ & $2(18,18)$ \\
DRGE & $1(9,09)$ & $1(9,09)$ & $1(9,09)$ & $0(0)$ \\
Asma & $1(9,09)$ & $0(0)$ & $2(18,18)$ & $0(0)$ \\
Hipotireoidismo & $0(0)$ & $0(0)$ & $1(9,09)$ & $0(0)$ \\
Esclerose múltipla & $0(0)$ & $0(0)$ & $2(3,92)$ & $1(9,09)$ \\
\hline
\end{tabular}

Fonte: Sato CSL, et al., 2022.

\section{DISCUSSÃo}

Sabe-se que fatores socioeconômicos influenciam diretamente na prevalência de sintomas depressivos na população, podendo ser a causa ou a consequência do surgimento destes, em uma relação bidirecional (SILVEIRA EF, 2016). Nesse cenário, busca-se uma associação entre alguns fatores protetores e de risco e o surgimento desses sintomas depressivos nos estudantes.

Ao analisarmos a correlação entre os fatores socioeconômicos com o BDI-I, é possível verificar na amostra que em um total de 51 estudantes, aproximadamente 49\% apresentaram sintomas moderados a graves, observando nestes uma predominância no sexo feminino. Esses dados estão de acordo com o estudo realizado por Lamônica LC (2019), com 96 estudantes concluintes do ensino médio que iriam prestar vestibular no Estado do Rio de Janeiro, no qual encontrou-se uma prevalência de sintomas depressivos entre estudantes do sexo feminino (38,54\%).

Literaturas demonstram que a prevalência de sintomas depressivos em mulheres, já a partir da adolescência, é o dobro quando comparada aos homens, o que pode ser observado na metanálise de Rombaldi AJ, et al. (2010). Dentre os motivos associados, destacam-se as questões socioculturais relacionadas à maior vulnerabilidade, à discriminação de gênero, à violência doméstica sexual e reprodutiva, além das diferenças fisiológicas e hormonais (JUSTOS LP e CALIL HM, 2006; RABELO IVM e ARAUJO MF, 2008).

Paralelamente a isso notou-se que grande parte dos entrevistados eram solteiros, o que se assemelha aos achados de Terra DHP, et al. (2013) que encontraram uma prevalência de $94,1 \%$ de solteiros entre os estudantes vestibulandos. Foi possível observar ainda no atual estudo que todos os casos de depressão moderada a grave foram provenientes deste grupo. Segundo Mesquita AM, et al. (2016), indivíduos solteiros estão mais vulneráveis ao desenvolvimento de sentimentos de solidão e abandono, o que pode acarretar em sintomas depressivos. Por outro lado, outros estudos demonstram uma relação inversa ao se associar depressão e o estado civil solteiro, principalmente nas faixas etárias mais jovens, mas afirma que por se tratar de uma doença com caráter emocional, sintomas depressivos podem ser influenciados pela qualidade e estabilidade dos relacionamentos (SILVEIRA EF, 2016)

No que refere ao local de residência e procedência, observou-se que poucos estudantes eram provenientes de outros municípios, no entanto metade destes apresentaram sintomas depressivos de moderado a grave. Esse fator mostra-se importante, uma vez que índices mais elevados de estresse, ansiedade e depressão têm sido identificados em estudantes que mudaram de cidade para estudar, 
principalmente naqueles que precisaram sair da casa dos pais. Tal fato possivelmente está relacionado à dificuldade de adaptação à nova realidade, associada à separação dos pais e à necessidade de um rápido amadurecimento pessoal, em uma transição do mundo infantil para o adulto (VIZZOTTO MM, et al., 2017).

Muitos indivíduos declaram-se religiosos nesta pesquisa. Acredita-se que a religião se apresenta como um fator protetor ao surgimento de sintomas depressivos, visto que o indivíduo é inserido em um ciclo de apoio social forte e estruturado, baseado em crenças comuns, o que se mostra extremamente importante para a criação de vínculos, para o fortalecimento do sentimento de pertencimento e do bem-estar, além de permitir o desenvolvimento de mecanismos de proteção contra o estresse diário (NASCIMENTO A e NASCIMENTO MS, 2018).

No entanto, isso não foi observado no presente estudo, no qual houve maior incidência de sintomas depressivos entre aqueles que possuíam uma religião, o que destoa do estudo de Melo CMl, et al. (2021), realizado com 161 alunos de cursos preparatórios do município de Aracaju/SE, o qual mostrou que $60 \%$ dos estudantes que não possuíam religião tinham grau de depressão de moderado a grave. Além disso, os autores também verificaram melhor qualidade de vida entre aqueles que possuíam práticas religiosas.

A renda familiar mensal também é um importante fator a ser considerado. Entre os entrevistados houve prevalência da renda familiar de média a alta, similar ao encontrado no estudo de Calixto FGC, et al. (2020), realizado com 170 alunos vestibulando de uma escola privada de ensino médio no Município de Sobral - CE, onde $91,2 \%$ dos estudantes apresentaram renda financeira de média a alta. Além disso, observou-se que todos os entrevistados que declararam possuir renda familiar de até 1 salário mínimo apresentaram graus de depressão de moderado a grave, o que está de acordo com o observado por Terra DHP, et al. (2013) o qual af irma que vestibulandos de menor renda apresentam maiores níveis de ansiedade e depressão, relacionado a preocupação e dificuldade de manutenção no curso pré-vestibular assim como maior tempo para entrada no mercado de trabalho.

Sabe-se que uma maior renda familiar é um fator protetor, uma vez que indivíduos com baixa condição socioeconômica estão mais vulneráveis o desenvolvimento de quadros depressivos, o que está associado principalmente à insegurança quanto ao futuro, ao maior estresse emocional, à baixa autoestima e à falta de acesso a um sistema de saúde de qualidade (SILVEIRA EF, 2016).

Verificou-se, neste estudo, que a maioria dos estudantes se declarou pardo, sendo também estre grupo o que mais apresentou sintomas depressivos de leve a grave. Esses dados não estão de acordo com o encontrado pelo IBGE (2014), que ao analisar o risco de depressão relacionada a cor/etnia no Brasil, notou uma maior incidência de sintomas depressivos entre pessoas brancas. No entanto, é necessária que essa análise seja feita considerando, também, o contexto socioeconômico, cultural e político no qual o sujeito está inserido (GONÇALVES AMC, et al., 2018; LAGUARDIA J, 2007).

Ao analisar a associação entre escolaridade e surgimento do transtorno depressivo maior, identifica-se que indivíduos que possuem um nível de escolaridade menor estão mais vulneráveis. Estudos realizados em território nacional demonstraram que tal fato é ainda mais significativo nas mulheres de baixa renda e com baixo nível de escolaridade. Ademais, os transtornos mentais, incluindo o transtorno depressivo, estão associados a maiores riscos de evasão escolar, menor rendimento escolar e dificuldade em concluir os estudos entre os jovens (CARPENA MX, et al., 2019; ALMEIDA-FILHO N, 2004).

No estudo de Melo CMI, et al. (2021), verificou-se uma correlação direta entre o índice de depressão e o tempo de realização de cursinhos pré-vestibulares, sendo mais prevalentes naqueles que prestariam vestibular para medicina (42\%) e que já tentavam o ingresso no curso a dois ou mais anos. $O$ atual estudo mostra-se em sintonia com este, verificando-se que dos 25 indivíduos que apresentaram sintomas depressivos de moderado a grave, $72 \%$ estavam a mais de 2 anos no curso pré-vestibular.

Além disso, quase a totalidade dos pesquisados informaram possuir apoio familiar nos estudos. $O$ apoio familiar configura-se como um importante fator de proteção, pois além de oferecer suporte psicológico e emocional ao jovem, contribui para o fortalecimento da sua autoestima e bem-estar, assim como para a redução do estresse e da autocrítica (CARDOSO HF, et al., 2018). Paralelamente a isso, pesquisas apontam 
que quanto maior o grau de depressão, menor será a percepção que o indivíduo terá do suporte familiar, o que pode contribuir negativamente para o quadro depressivo (BAPTISTA MN e OLIVEIRA AA, 2004).

Ao analisar a relação entre as comorbidades prévias relatadas pelos participantes e suas pontuações no BDI-I, torna-se necessário pontuar a possibilidade de tais patologias estarem influenciando, direta ou indiretamente, nos estados depressivos apresentados. Dentre os participantes que relataram atopias, houve divergências quanto ao grau de depressão segundo o BDI-I apresentado. Isto indica que existem outros fatores, além da patologia alérgica, que contribuem para o estado emocional do participante. Bedolla-Barajas $\mathrm{M}$, et al. (2017) demonstraram que, ainda que haja correlação entre atopias e a depressão, não há associação entre o processo alérgico e o grau moderado ou severo do transtorno, o que condiz com os resultados encontrados no presente estudo.

Ademais, nesse artigo, foram encontrados dois pacientes asmáticos com pontuação do BDI-I compatível com depressão moderada. Kuehn BM, et al. (2008) demonstraram que pacientes asmáticos, principalm ente aqueles com asma não compensada, são mais propensos a desenvolver distúrbios de humor, incluindo 0 Transtorno Depressivo Maior, resultados condizentes com os dados encontrados nessa pesquisa.

Como comprovado por Orozco RIM, et al. (1985), o hipotireoidismo clínico pode se demonstrar como fator de risco para o desenvolvimento do Transtorno Depressivo Maior sendo corroborado por Oliveira MC, et al. (2001), os quais encontraram, dentre sua amostra, ao menos 3 sintomas depressivos em $29,3 \%$ e depressão diagnosticada por especialista em 12,8\%, sendo a amostra total de 117 pacientes hipotireóideos. Logo, a relação entre hipotireoidismo e o grau moderado de depressão acusado pela pontuação do BDI-I mostra-se condizente com a literatura.

A Esclerose Múltipla (EM) é uma doença crônica e inflamatória com repercussão sistêmica e que gera danos ao sistema nervoso central, causando déficits motores, sensitivos e cognitivos nos acometidos. Patten SB, et al. (2003) e Chwastiak L, et al. (2002) demonstraram a alta prevalência de sintomas depressivos em pacientes diagnosticados com EM, o que corrobora os dados encontrados nesse estudo, no qual a participante com esclerose múltipla pontuou o equivalente a sintomatologia de depressão severa pelo BDI-I.

Todas as doenças relatadas durante a realização dessa pesquisa são consideradas doenças crônicas, as quais são fator de risco para surgimento de sintomas depressivos. Boing AF, et al. (2012), em um estudo transversal de base populacional, demonstraram clara associação entre doenças crônicas e sintomas depressivos, estando presente em $16 \%$ da amostra. Os resultados encontrados nesta pesquisa condizem com a literatura atual, na qual $13,72 \%$ da amostra possui concomitantemente, doenças crônicas e grau moderado ou severo de sintomas depressivos.

\section{CONCLUSÃO}

Percebeu-se no estudo que a incidência de sintomas depressivos entre os estudantes de pré-vestibular é significativamente alta, atingindo principalmente mulheres jovens, pardas, solteiras e de classe média, que estavam a mais de 2 anos prestando vestibular. A alta frequência de quadros depressivos entre jovens é um preocupante problema de saúde pública, no entanto, é possível notar que ainda há uma deficiência de produção científica abordando a depressão entre vestibulandos, tendo sido esta uma das principais dificuldades encontradas no desenvolvimento do estudo. Logo, nota-se que ainda há certa invisibilidade e estigma em torno da temática da depressão entre os estudantes pré-universitários, sendo alguns de seus sintomas como tristeza, estresse e baixa autoestima, vistos como algo inerente a essa etapa que antecipa o vestibular. Diante disso, ressalta-se a necessidade do desenvolvimento de futuras pesquisas e 0 aprofundamento do debate sobre a problemática no meio educacional. Espera-se com os resultados encontrados chamar a atenção das instituições de ensino aos fatores de risco e aos sinais indicativos de quadros depressivos entre seus alunos, para que busquem estratégias de promoção da saúde mental e qualidade de vida, of erecendo apoio psicológico contínuo. 


\section{REFERÊNCIAS}

1. ALMEIDA-FILHO N, et al. Social inequality and depressive disorders in Bahia, Brazil: interactions of gender, ethnicity, and social class. Soc Sci Med, 2004;59(7): 1339-1353.

2. BAHLS SC, BAHLS FRC. Depressão na adolescência: características clínicas. Interação em Psicologia, 2002;6(1): 49-57.

3. BAPTISTA MN, OLIVEIRA AA. Sintomatologia de depressão e suporte familiar em adolescentes: um estudo de correlação. Rev Bras Cresc Desenv Hum, 2004; 14(3):53-59.

4. BEDOLLA-BARAJAS M, et al. Rinite como fator associado para ansiedade e depressão entre adultos. Brazilian Journal of Otorhinolaryngology, 2017;83(4):432-438.

5. BIAZUS CB, RAMIRES VRR. Depressão na adolescência: uma problemática dos vínculos. Psicol Estud, 2012;17(1): 83-91.

6. BOING AF, etal. Associação entre depressão e doenças crônicas:um estudo populacional. Rev Saúde Pública, 2012; 46(4):617-623.

1. CALIXTO FGC, et al. O pré-vestibular como fator desencadeador de depressão em estudantes da rede privada. Castro, Paula Almeida de. (org.) de Avaliação:Processos e Políticas Cam pina Grande: Realize eventos, 2020.16 p.

2. CARDOSO HF, et al. Indicadores de saúde mental em jovens: fatores de risco e de proteção. Est Inter Psicol, 2018 ; 9(3): 3-25.

3. CARPENA MX, et al. Sociodemographic, behavioral, and health-related risk factors for depression among men and women in a southern Brazilian city. Braz J Psychiatry, 2019; 41(5):396-402.

4. CHWASTIAK L, et al. Depressive symptoms and severity of illness in multiple sclerosis: epidemiologic study of a large community sample. Am J Psychiatry, 2002; 159: 1862-1868

5. GONÇALVES AMC, et al. Prevalência de depressão e fatores associados em mulheres antedidade pela Estratégia Saúde da Família. J Bras Psiquiatr, 2018;67(2): 101-109.

6. INSTITUTO BRASILEIRO DE GEOGRAFIA E ESTATÍSTICA (IBGE). Pesquisa nacional de saúde 2013: percepção do estado de saúde, estilos de vida e doenças crônicas: Brasil, grandes regiões e unidades da federação. 2014. Disponível em: https://biblioteca.ibge.gov.br/visualizacao/livros/liv91110.pdf. Acessado em:20 de setembro de 2021.

7. JUSTO LP, CALIL HM. Depressão: o mesmo acometimento para homens e mulheres?. Rev Psiquiatr Clín, 2006; 33(2): 74-79.

8. KUEHHBM. Asthma linked to psychiatric disorders. JAMA, 2008;299(2): 158-60.

9. LAGUARDIA J. Raça e epidemiologia: as estratégias para construção de diferenças biológicas. Cien Saude Coletiva, 2007; 12(1): 253-261.

10. LAMỐNICA LC. Prevalência de indicadores de ansiedade, estresse e depressão entre adolescentes vestibulandos concluintes do ensino médio. 2019. 35 f. Trabalho de Conclusão de Curso (Bacharelado em Psicologia) - Instituto de Ciências da Sociedade e Desenvolvimento Regional. Universidade Federal Fluminense, Campos dos Goytacazes, 2019

11. LIMA GMPA, et al. Aspectos psicossociais e intervenções realizadas com o adolescente depressivo: revisão integrativa nacional da última década. Psi Divers Saúde, 2018;7(3): 453-462.

12. LIPP MEN. Mecanismos neuropsicológicos do stress: teoria e aplicações clínicas. São Paulo: Casa do Psicólogo; 2003.227p.

13. MELO CMI, et al. Ansiedade em alunos de pré-vestibular da cidade de Aracaju Anxiety in pre college admission test students in the city of Aracaju. Brazilian Journal of Health Review, 2021;4(3):12470-12480.

14. MESQUITA AM, et al. Depressão em estudantes de cursos da área da saúde de uma universidade em Mato Grosso. $\mathrm{J}$ Health NPEPS, 2016;1(2): 2018-230.

15. MINISTÉRIO DA SAÚDE. Proteger e cuidar da saúde de adolescentes na atenção básica. Brasília-DF:Brasil, 2017. Disponível em: https://bvsms.saude.gov.br/bvs/publicacoes/proteger_cuidar_adolescentes_atencao_basica.pdf. Acessado em: 9 de setembro de 2021.

16. NASCIMENTO A, NASCIMENTO MS. Associação entre religiosidade e depressão: uma análise histórica e de resultados na psicologia. Revista de estudos e pesquisa da religião, 2018;21(2):145-152.

17. OLIVEIRA MC, et al. Sinais e sintomas sugestivos de depressão em adultos com hipotireoidismo primário. Arq Brasil Endocrinol Metab, 2001;45(6): 70-575.

18. ORGANIZAÇÃO PAN-AMERICANA DE SAÚDE (OPAS). Folha informativa: Depressão. 2018a. Disponível em: https://www.paho.org/bra/index.php?option=com_content\&view=article\&id=5635:folha -informativadepressao\&ltemid=822. Acessado em: 19 de setembro de 2021.

19. ORGANIZAÇÃO PAN-AMERICANA DE SAÚDE (OPAS). Folha informativa: Saúde mental dos adolescentes. $2018 \mathrm{~b}$. Disponível em: https://www.paho.org/bra/index.php?option=com_content\&view=article\&id=5779:folha-informativasaude-mental-dos-adolescentes\&ltemid=839. Acessado em: 18 de setembro de 2021 .

20. OROZCO RIM. Presencia de depresión en pacientes hipotiroideos. Salud Mental, 1985;8: 90-93.

21. PATTEN SB, et al. Major depression in multiple sclerosis: a population based perspective. Neurology, 2003; 61:15241527.

22. RABELO IVM, ARAUJO MF. Violência de gênero na perspectiva da saúde mental. Rev Psicol. UNESP, 2008;7(1): 123-132.

23. ROMBALDI AJ, et al. Prevalência e fatores associados a sintomas depressivos em adultos do sul do Brasil: estudo transversal de base populacional. Rev Bras Epidemiol, 2010;13(4):620-629.

24. SANTOS MA, et al. Sintomas psicológicos relacionados ao stress em pré-vestibulandos. Anais da $18^{\underline{a}}$ Semana de Pesquisa da Universidade Tiradentes, 2016.

25. SCHNEIDERACN, RAMIRES VRR. Vínculo parental e rede de apoio social: relação com a sintomatologia depressiva na adolescência. Aletheia, 2007;(26):95-108.

26. SILVEIRA EF. Fatores socioeconômicos e psicossociais relacionados à prevalência da depressão no Brasil. Dissertação. Universidade Federal do Rio Grande do Sul, Porto Alegre, 2016.

27. TERRA DHP, et al. Ansiedade e depressão em vestibulandos. Odontologia Clínico-Científica, 2013;12(4):273-276.

28. VIZZOTTO MM, et al. Saudades de casa: indicativos de depressão, ansiedade, qualidade de vida e adaptação de estudantes universitários. Rev Psicol Saúde, 2017;9(1):59-73.

29. WORLD HEALTH ORGANIZATION (WHO). Mental health action plan 2013-2020. 2013. Disponível em: https://www.who.int/publications/i/item/9789241506021. Acessado em:18 de setembro de 2021. 